\title{
“MERAKI, DE NIÑOS PARA NIÑOS”, UNA EXPERIENCIA DE APRENDIZAJE SERVICIO SOLIDARIO (APSS)
}

\section{“MERAKI, FROM CHILDREN TO CHILDREN", AN EXPERIENCE OF LEARNING SOLIDARITY SERVICE (APSS)}

\section{Pedro Antonio García Tudela}

\begin{abstract}
Resumen
Son numerosas las metodologías, estrategias y recursos con los que contamos hoy día para desarrollar una educación integral, de calidad y competente para el alumnado. En el presente trabajo se explicita una experiencia basada en un proyecto de Aprendizaje Servicio Solidario (ApSS) a beneficio de AFACMUR (Asociación de Familiares de niños con Cáncer de Murcia) desarrollada por un colegio público de una localidad de Murcia, concretamente se ha llevado a cabo con todos los escolares del nivel de Educación Primaria.

El proyecto que aquí se muestra tiene la finalidad principal de desarrollar una educación holística del alumnado, la cual está sustentada sobre una base teórica,
\end{abstract}


práctica y actitudinal. Teniendo esta el fin principal de formar alumnos competentes para la vida y comprometidos con la sociedad.

Los resultados que se extraen de esta experiencia evidencian el logro de los objetivos planteados e incluso superan la expectativa que se tenía de "Meraki, de niños para niños".

\section{Palabras Clave}

Aprendizaje Servicio Solidario, AFACMUR (Asociación de Familiares de niños con Cáncer de Murcia), educación holística, "Meraki, de niños para niños" y comunidad educativa.

\section{Abstract}

There are several methodologies, strategies and resources that we can use today to develop a comprehensive, quality and competent education for students. The present study explains an experience based on a project of solidarity service learning $(S L)$ in aid of AFACMUR (Association of relatives of children with Cancer from Murcia) developed by a public school from a locality of Murcia, specifically, it was carried out with all primary school students.

This project has the main purpose of developing a holistic education of students; it is based on a theoretical, practical and attitudinal basis. And it has the main purpose of developing competent students for life and committed to society.

The results that are extracted show the achievement of the objectives and even exceed the expectation of "Meraki, from children to children".

\section{Key Words}

Servide learning, AFACMUR (Association of relatives of children with Cancer from Murcia), holistic education, "Meraki, from children to children" and educative community. 


\section{Fundamentación teórica sobre el Aprendizaje Servicio}

Indiferentemente del centro escolar que se trate, la finalidad que persigue cualquier colegio es la de guiar y formar de manera holística a la persona, para que de esta forma pueda ser un ciudadano competente para la vida. Dicho principio ya era señalado en el siglo XX por la escuela Decroly, concretamente afirmaba que se debe educar para la vida a través de la vida misma, conllevando el alcance de dicha meta una serie de retos que a día de hoy siguen debatiéndose. Pero para desarrollar dicho aprendizaje deseable, es necesario partir del aprender a: ser, a convivir, a participar y a habitar en el mundo. A todo ello se hace alusión en Puig Rovira, 2011. De esta forma estaremos aprendiendo a convivir junto a unos deberes y unos derechos esenciales que garanticen una sociedad democrática, plural y participativa, además de estar dotados de unos valores éticos universales.

Para poder educar en justicia, solidaridad, equidad social, etc., de una manera significativa hay que partir de la realidad para valorar situaciones de desigualdad y necesidad como bien es señalado en Ferrán y Guinot (2012). El reconocimiento y conocimiento de ello posibilitará una mayor concienciación, implicación y compromiso del alumnado, ya que ellos pueden ser los protagonistas de las acciones que mejoren la situación estudiada.

Diversas opciones son las que tenemos hoy día para poder acercarnos al desarrollo de una educación integral en nuestras aulas, ejemplo de ello son las metodologías activas; el trabajo de la Inteligencia Emocional y Social; la inclusión de las TAC (Tecnologías del Aprendizaje y el Conocimiento); el aprendizaje basado en proyecto $(A B P)$, entre otras.

En Rodríguez y Ordóñez (2015), se explicita que una opción para que el alumno aprenda de manera activa, la cual valora tanto la vertiente académica como el desarrollo de competencias profesionales, es el Aprendizaje Servicio (ApS). Esta forma de aprendizaje la cual se suele desarrollar en proyectos concretos a nivel de aula, tramo o colegio se sustenta principalmente en adquirir el compromiso de aportar un servicio a la comunidad. 


\section{1. ¿Qué es el Aprendizaje Servicio?}

Son numerosas las definiciones que se han aportado del aprendizaje servicio, entre ellas destacar la de Gil (2012), este autor señala que el ApS es una metodología que pretende alcanzar el aprendizaje de unos determinados contenidos académicos al mismo tiempo que se presta un servicio a la comunidad. Este autor añade que es una metodología que se relaciona plenamente con el aprendizaje experimental, ya que pretende dar a este una significatividad. Para desarrollar un proyecto de ApS de manera adecuada se tiene que considerar al alumno como el centro de dicho proceso, teniendo el docente el rol de guía, por lo que tendrá que plantear tareas y los alumnos han de ir realizándolas y superando todos los problemas que se puedan presentar aplicando el pensamiento estratégico.

Murphy (2010) y Verjee (2010) afirman que la adecuada puesta en práctica de un proyecto de ApS desarrolla de manera óptima las capacidades intelectuales y las habilidades profesionales del alumnado.

Algunos autores señalan la formación holística que ofrece al alumnado este tipo de aprendizaje, definiéndolo como una pedagogía o filosofía de la Educación (Stanton, 1990). Una definición que refleja este hecho es la siguiente: "El aprendizaje Servicio (...) es también una filosofía de crecimiento humano y sentido, una visión social, un modo de aproximación hacia la comunidad y una manera de conocer." (Kendall, 1990, p. 23).

\subsection{Beneficios del Aprendizaje Servicio}

Diversos trabajos (Conway, Amel y Gerwien, 2009; Murphy, 2010 y Verjee, 2010) son los que hacen alusión a los beneficios de dicha metodología de trabajo, algunos aspectos de los que señalan evidenciados con sus investigaciones son: mejora la vertiente social, debido a que los participantes del proyecto han de cooperar, dialogar, tomar decisiones, etc.

En líneas similares, Rubio (2009) destaca que el ApS desarrolla tanto competencias sociales como intrapersonales, así como el pensamiento cívico, la ciudadanía, la transformación social, entre otros aspectos.

Martínez (2014) opta por concretar aun más los beneficios que tiene esta metodología, algunos de estos son: desarrollo de capacidades necesarias (evaluar, 
ejecutar, corregir, entre otras); brindar la ocasión para que los estudiantes descubran intereses y vocaciones; desarrollar actitudes pro-sociales y de resiliencia, ya que han de saber cómo afrontar las adversidades, etc.

La reciprocidad que conlleva un proyecto de ApS también es un hecho destacable, en Ferrán y Guinot (2012) se hace alusión a la reciprocidad que se da entre el aprendizaje y el servicio. Ello es debido a que el primero mejora el servicio, ya que el aprendizaje se manifestará en forma de aplicación práctica a la realidad (a la comunidad), teniendo como resultado un servicio de calidad. Por otro lado, el servicio mejora también el aprendizaje, ya que está evidenciando con la práctica lo que se está aprendiendo, además de motivar al alumnado y otorgar un mayor sentido a los contenidos trabajados. Siendo el aprendizaje significativo uno de los principales beneficios de esta metodología.

\subsection{Finalidades del Aprendizaje servicio y focos de implementación.}

El objetivo que siempre se persigue es ofrecer un servicio a la comunidad, bien sea vinculado a alguna asociación; a la naturaleza; mejorar o arreglar alguna instalación urbanística, etc. "La juventud asume roles significativos y desafiantes en una variedad de lugares, tales como: guarderías, museos (...) o centros de jubilados. Las actividades en estos lugares pueden incluir lectura a niños (...), guía de museos, limpieza de barrios, etc." (Halsted, 1998, p. 23-24).

Puig, Batlle, Bosch y Palos (2007) explicitan las diferentes finalidades que se persiguen con el desarrollo de un proyecto de aprendizaje servicio, entre ellas destacar: desarrollar el pensamiento crítico y reflexivo; valorar y ser sensible ante las necesidades de la comunidad; afianzar el compromiso social; ser responsable cívicamente y trabajar aspectos académicos.

Halsted (1998), señala que el ApS es una propuesta educativa que se puede llevar a cabo tanto en contextos educativos formales como no formales. Así como con cualquier curso, tan solo hay que concretar el proyecto al nivel académico y a la realidad del alumnado. Además, no solo es algo que ha de desarrollarse entre alumnado y profesorado, como bien señala Martínez (2014), una experiencia de ApS se puede y se debe abrir al alumnado, padres, profesorado y a todos los agentes que se pudiesen implicar (voluntariado, entidades, gestores locales, etc.). Aunque no se ha 
delegar todas las responsabilidades educativas en diversos agentes, ya que como bien afirma Martínez y Ruíz (1996), el docente es una de las principales personas que tiene como función guiar al alumnado en el camino más adecuado dependiendo de su realidad para poder alcanzar de esta forma la madurez y así afrontar de manera adecuada las adversidades y toda situación que se le plantee en la vida.

\subsection{Requisitos para la puesta en práctica del ApS y "triángulo EMO"}

Cabe destacar que son varias las publicaciones (Puig, 2009; Tapia, 2004, 2007, 2013), las que señalan requisitos que han de estar presentes para poder considerar una propuesta educativa como de aprendizaje servicio. Principalmente, todas coinciden en los siguientes ítems:

- Deben plantearse y trabajarse aprendizajes relacionados con el currículum escolar.

- El aprendizaje debe estar insertado en un servicio a beneficio de la comunidad.

- Debe existir un protagonismo de los escolares.

Para desarrollar el proyecto "Meraki, de niños para niños", se han tenido en cuenta los puntos anteriores, pero se ha desarrollado también una figura esencial que tiene la finalidad de reagrupar, sintetizar y ofrecer de una manera más visual y por lo tanto más legible los puntos clave con los que ha de contar un proyecto educativo para que pueda ser denominado como aprendizaje servicio. Esta figura titulada "triángulo EMO" tiene su origen en los estudios recién señalados de Puig y Tapia. A continuación, se puede apreciar en la figura 1. 


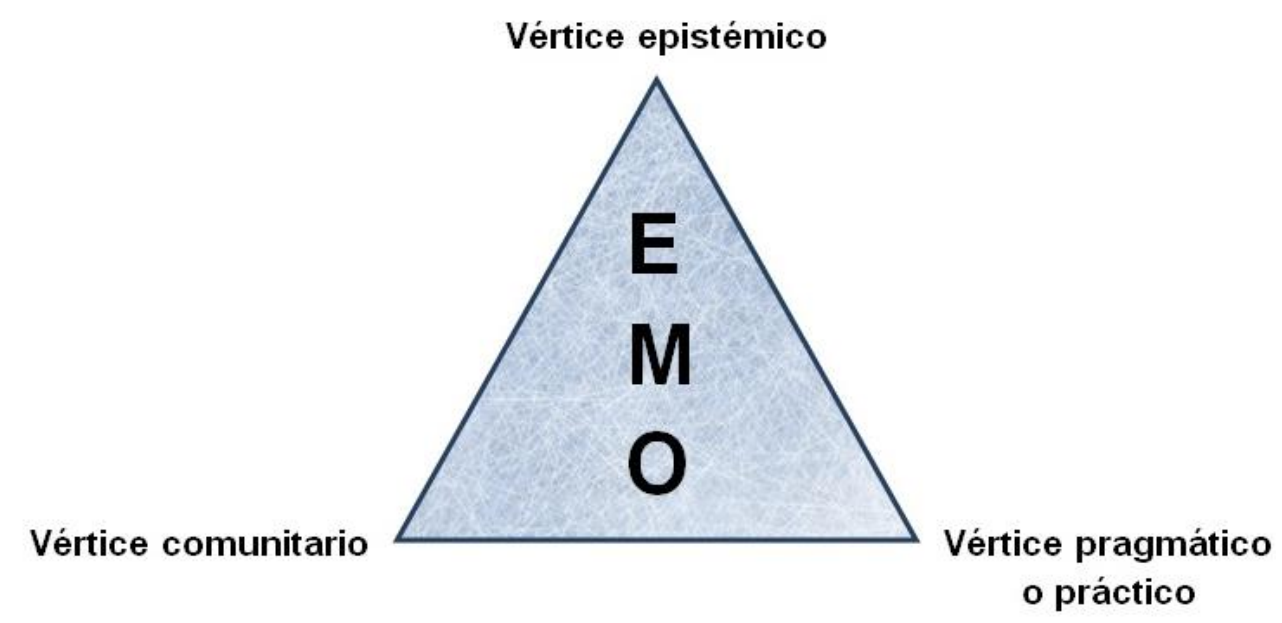

Figura 1. Triángulo EMO. Fuente: elaboración propia

Los tres puntos esenciales que ha de contener un proyecto de aprendizaje servicio para que sea considerado como tal coinciden con los tres vértices de un triángulo equilátero. De una manera más detallada serían los siguientes:

- Vértice epistémico. Este punto se corresponde con los contenidos de una o diferentes áreas curriculares, ya que un proyecto de aprendizaje servicio ha de estar justificado con unos contenidos acordes al curso con el que se esté desarrollando la experiencia. De esta manera, no se quedará en una actividad aislada de solidaridad o de servicio a la comunidad, sino que tendrá un trasfondo. Razón de ello no es solo por el reflejo de unos contenidos curriculares, también porque el vértice epistémico hace alusión a la educación en valores que hay que planificar en la organización del proyecto, recogiendo qué valores y cómo los vamos a trabajar en el aula.

- Vértice pragmático o práctico. Este hace referencia al desarrollo de las competencias que desarrollará el alumnado en pro del alcance de los objetivos prefijados, tanto a nivel conceptual, práctico y actitudinal. Es decir, son las actividades preparadas por el docente, las cuales han de estar orientadas a trabajar las competencias fijadas en el informe de desarrollo del proyecto de aprendizaje servicio.

- Vértice comunitario. Uno de los puntos clave para que el proyecto no quede en una aislada educación en valores o en una actividad de solidaridad, es justificar el vértice epistémico y el pragmático con uno o varios objetivos que promuevan la mejora 
de las condiciones de vida de las personas y el entorno.

Si en el informe de planificación del proyecto de ApS se tienen en cuenta los tres vértices anteriores y una adecuada temporalización, organización del grupo o de las clases participantes, así como una colaboración en medida de lo posible de la comunidad educativa, se desarrollará un óptimo proyecto, el cual tendrá como principal origen y final la emoción y la motivación.

\section{Objetivos del presente trabajo}

- Presentar a los profesionales una iniciativa y una experiencia deseable de llevar a cabo en otros centros educativos.

- Reflexionar sobre el desarrollo y los resultados de este proyecto de Aprendizaje Servicio.

\section{Proyecto de Aprendizaje Servicio «Meraki, de niños para niños »}

\subsection{Presentación}

El presente proyecto de Aprendizaje Servicio Solidario ("Meraki, de niños para niños") se ha llevado a cabo en un colegio de entidad pública en Totana (Murcia), cabe destacar que ha tenido una duración aproximada de dos meses, correspondiéndose estos con el tercer trimestre del curso académico 2016/2017.

Se ha llevado a cabo con todos los escolares pertenecientes a la etapa de Educación Primaria, por lo que ha tenido una participación total de 187 estudiantes. Siendo el tutor de cada grupo el encargado de adaptar las líneas de actuaciones generales para todo el centro a su contexto particular de aula. Por lo que en cada clase se ha trabajado con una metodología y unos materiales diferentes.

Señalar que los cursos pertenecientes a Educación Infantil solo han participado en una de las actividades previstas (la grabación del videoclip).

En el apartado de aplicación se detallará de manera más exhaustiva la temporalización, organización y actividades desarrolladas en dicho proyecto de ApS. No obstante, en este punto se ha de hacer alusión a que el servicio previsto y presentado a la comunidad educativa, es decir, la finalidad era conseguir hacer una donación económica y anímica a los niños enfermos de oncología a través de 
AFACMUR (Asociación de Familiares de niños con Cáncer de Murcia).

\subsection{Objetivos del proyecto}

El objetivo principal que se planteó con «Meraki, de niños para niños» fue:

- Desarrollar un proyecto de Aprendizaje Servicio Solidario que refleje principalmente contenidos del área de Ciencias de la Naturaleza.

Otros objetivos que también fueron planteados son los siguientes:

- Formar a alumnos competentes para la vida y comprometidos con la sociedad.

- Informar y concienciar al alumnado sobre la oncología infantil.

- Conseguir hacer dos donaciones a AFACMUR. Por un lado, fondos económicos para la asociación y por otro, una donación anímica a través de un videoclip que anime a todos los niños oncológicos.

\subsection{Contexto de aplicación}

A nivel general, el colegio donde se ha desarrollado dicha experiencia de aprendizaje servicio se ubica en el extrarradio de Totana (Murcia). Es un barrio compuesto por familias con un nivel socioeconómico bajo, siendo la ocupación laboral predominante la perteneciente al sector primario. Además, una gran cantidad de escolares provienen de pedanías cercanas, por lo que muchos de ellos solo coinciden en horas lectivas.

La atención a la diversidad es una realidad latente en dicho centro escolar, ya que cada grupo cuenta con una alta proporción de alumnos inmigrantes, principalmente árabes y latinoamericanos. Muchos de ellos presentando diversas dificultades de aprendizaje debido a las constantes incorporaciones tardías al sistema educativo. Además, este colegio cuenta con el apoyo diario de una profesional de audición y lenguaje y un pedagogo terapéutico. Los cuales trabajan diariamente con el alumnado que presenta dificultades con el fin de paliar dicha problemática.

En la actualidad, este barrio sigue creciendo con la construcción de nuevas viviendas debido a la llegada en su mayoría de nuevas familias sudamericanas. 
Se detecta escasez de algunos servicios en el barrio donde se ubica el colegio, como farmacias y entidades bancarias. Hasta hace aproximadamente 3 meses los vecinos se tenían que desplazar hasta el único centro de salud con el que contaba la ciudad. Ahora, cuentan con uno nuevo en este barrio.

\subsection{Organigrama y desarrollo del proyecto}

Para conocer la manera de la que se ha organizado "Meraki, de niños para niños" y cómo se ha aplicado en las aulas. Se ha de partir del organigrama que se ha tenido en cuenta, este se puede apreciar en la tabla 1:

\begin{tabular}{|c|c|}
\hline $\begin{array}{l}\text { Primera semana } \\
\text { de mayo de } 2017 \\
\text { en CCP } \\
\text { (Comisión de } \\
\text { coordinación } \\
\text { pedagógica) y } \\
\text { claustro ordinario }\end{array}$ & $\begin{array}{l}\text { Explicación por parte del coordinador del proyecto al } \\
\text { personal docente la propuesta de llevar a cabo una } \\
\text { experiencia de Aprendizaje Servicio Solidario en el } \\
\text { colegio. Especificando en esta reunión las diferentes } \\
\text { fases con la que cuenta el proyecto y las dos } \\
\text { actividades finales: grabación de un videoclip para } \\
\text { apoyar a todos los niños que están en la unidad de } \\
\text { oncología infantil del hospital Virgen de la Arrixaca } \\
\text { (Murcia) y recaudación de fondos económicos. }\end{array}$ \\
\hline $\begin{array}{l}23 \text { y } 24 \text { de mayo de } \\
2017\end{array}$ & $\begin{array}{l}\text { "AfacTour": Ruta por las diferentes clases de la etapa de } \\
\text { Educación Primaria presentado a través de un Prezi qué } \\
\text { es la oncología infantil; AFACMUR; Efecto Pigmalión y } \\
\text { cómo ayudaremos a la asociación y animaremos a todos } \\
\text { los niños. } \\
\text { Enlace } \quad \text { a } \quad \text { Prezi } \quad \text { "AfacTour": } \\
\text { https://prezi.com/a8ymg6izxwx0/afacmur-y-deitania/ }\end{array}$ \\
\hline $\begin{array}{l}\text { Del } 25 \text { al } 29 \text { de } \\
\text { mayo de } 2017\end{array}$ & $\begin{array}{l}\text { Trabajo independiente de cada grupo con su tutor/a. Lo } \\
\text { principal que se trabajará en las sesiones dedicadas a } \\
\text { este proyecto serán contenidos relacionados con las } \\
\text { Ciencias de la Naturaleza (qué es la célula, tipos de } \\
\text { enfermedades, tratamientos, etc.). Así como una }\end{array}$ \\
\hline
\end{tabular}




\begin{tabular}{|c|c|}
\hline & $\begin{array}{l}\text { educación en valores } \text { (solidaridad, voluntad, } \\
\text { generosidad, superación, etc.) }\end{array}$ \\
\hline $\begin{array}{l}29 \text { y } 30 \text { de mayo de } \\
2017\end{array}$ & $\begin{array}{l}\text { Montaje de un puesto solidario en el recreo en el cual se } \\
\text { venderán: pulseras solidarias de AFACMUR ( } 3 \\
€ / \text { pulsera) y entradas para el desfile que habrá el } 2 \text { de } \\
\text { junio de } 2017 \text { en Totana a beneficio de la asociación (3 } \\
€ / \text { entrada). } \\
\text { Los mismos alumnos serán los que venderán a otros } \\
\text { escolares, docentes y familias. }\end{array}$ \\
\hline $\begin{array}{l}\text { Hasta el } 6 \text { de junio } \\
\text { de } 2017\end{array}$ & $\begin{array}{l}\text { Cada clase ensaya independientemente la canción y el } \\
\text { fragmento que le corresponde para el videoclip. Así } \\
\text { como la elaboración del material y carteles que vayan a } \\
\text { utilizar el día de la grabación. }\end{array}$ \\
\hline $\begin{array}{l}6 \text { y } 7 \text { de junio de } \\
\qquad \begin{array}{l}2017 \\
\quad \text { Tabla 1. Ors }\end{array}\end{array}$ & $\begin{array}{l}\text { Grabación del videoclip y visita de la televisión regional } \\
(7 \mathrm{RM}) \text {. } \\
\text { Primero los fragmentos de cada clase. Por último, el lazo } \\
\text { dorado creado en el patio del colegio por parte de todos } \\
\text { atosłpardictpantlesde niños para niños". Fuente: elaboración propia }\end{array}$ \\
\hline $\begin{array}{l}\text { A partir del } 12 \text { de } \\
\text { junio de } 2017\end{array}$ & $\begin{array}{l}\text { Visionado y difusión del videoclip creado por el CEIP } \\
\text { Deitania a través de las redes sociales y de los medios } \\
\text { de comunicación locales y regionales. Donación del } \\
\text { dinero conseguido y reflexión sobre toda la experiencia } \\
\text { llevada a cabo. }\end{array}$ \\
\hline
\end{tabular}

Conociendo el organigrama en el que se basa este proyecto, ahora se desglosarán de una manera más detallada determinadas actividades señaladas anteriormente. 
El "AfacTour" se ha realizado por las diferentes clases de este centro educativo. La presentación Prezi que se utilizó fue la misma en todos los grupos, ya que era sencilla y permitía explicar en cada clase de una manera más personalizada y adaptada al nivel lo que se pretendía enseñar.

Cada sesión de esta ruta por las clases ha tenido una duración de unos 20-25 minutos aproximadamente, las cuales han sido impartidas por el coordinador del proyecto. Cada presentación del "AfacTour" se dividía en diferentes bloques, los cuales eran: base conceptual sobre qué es el cáncer, tratamientos, consecuencias, etc.; explicación de la oncología infantil; qué es AFACMUR; qué necesitan los niños/as oncológicos/as y por último, cómo vamos a desarrollar "Meraki, de niños para niños".

Una vez que cada grupo había recibido la sesión recién explicitada, era momento de trabajar con su tutor/a los contenidos referentes a la temática principal de este proyecto. Los contenidos que más se han trabajado dependiendo del curso han sido: la célula; sistema inmunológico; tipos de enfermedades (sintomatología, tratamientos, etc.), entre otros. La manera de la que han trabajado dichos contenidos ha sido diferente dependiendo del curso y del docente, ya que cada profesional tenía libertad para decidir los recursos, la metodología y la temporalización que estimase más óptima para su grupo. Principalmente, se han utilizado: vídeos didácticos; brainstorming; role play donde los alumnos se han convertido en doctores que tenían que diagnosticar a otros alumnos que adquirían el rol de pacientes, etc.

Por otro lado, periódicamente se ha desarrollado con cada curso una educación en valores para trabajar los que se habían prefijado en el informe del proyecto, los cuales fueron seleccionados por tener una relación directa con "Meraki, de niños para niños”. Estos fueron principalmente: solidaridad, generosidad, voluntad, superación, cooperación y empatía. Al igual que en el caso anterior de los contenidos teóricos, cada docente tuvo la libertad de elegir la manera de desarrollar esta educación en valores del alumnado. En este caso, los recursos más utilizados han sido: cortos de Disney sobre los que se han reflexionado tras su visionado; lectura de cuentos breves con la posterior realización de una hoja de comprensión y/o un debate; lectura de titulares y breves resúmenes de noticias sobre los que el alumnado identificaba los valores que estaban presentes, entre otros más. 
En referencia al puesto solidario que se montó dos días en el recreo del colegio, señalar que fueron determinados alumnos (seleccionados con un sorteo) del segundo tramo de Educación Primaria los que se encargaron de vender tanto las pulseras solidarias de AFACMUR, como las entradas para el evento solidario que se llevaría a cabo en Totana a beneficio de esta misma asociación. Las pulseras que se vendieron se pueden apreciar en la figura 2.

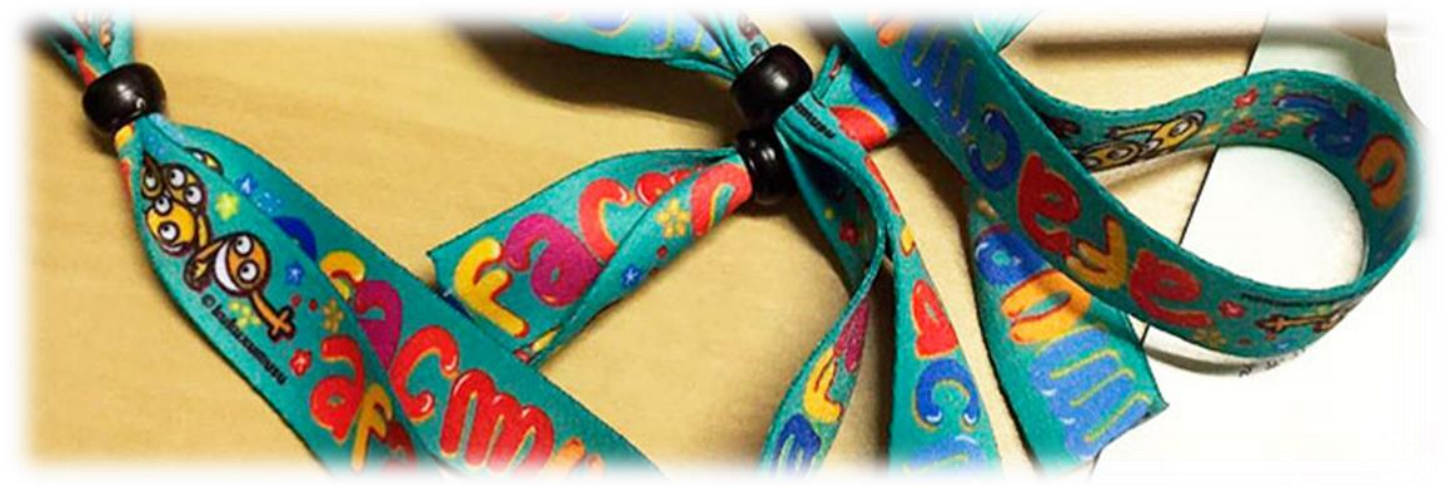

Figura 2. Pulseras de AFACMUR. Fuente: https://goo.gl/H3f2XR

Por último, para el rodaje del videoclip se segmentó equitativamente la canción de "Guerreros contra el miedo" (https://www.youtube.com/watch?v=aXRbEI3xYrl) en tantas partes como grupos participantes. Una vez que el alumnado fue informado del fragmento que les correspondía, comenzaron a aportar ideas sobre qué podían hacer (coreografía, vestuario, decoración, escenario, etc.). Además, cada grupo tuvo que pensar y elaborar un cartel con una frase breve que transmitiese ánimo, por lo que también era una tarea a realizar por parte de todo el grupo.

Una vez llegado el día de la grabación, se siguió un horario de rodaje elaborado para que así cada grupo supiese en qué momento de la jornada recibiría la visita de las cámaras para grabar su parte y estar preparados. Además, de esta forma, se pudo desarrollar el resto de la jornada lectiva con normalidad. Algunas fotos del proceso de grabación se pueden apreciar en las figuras 3, 4, 5 y 6 . 


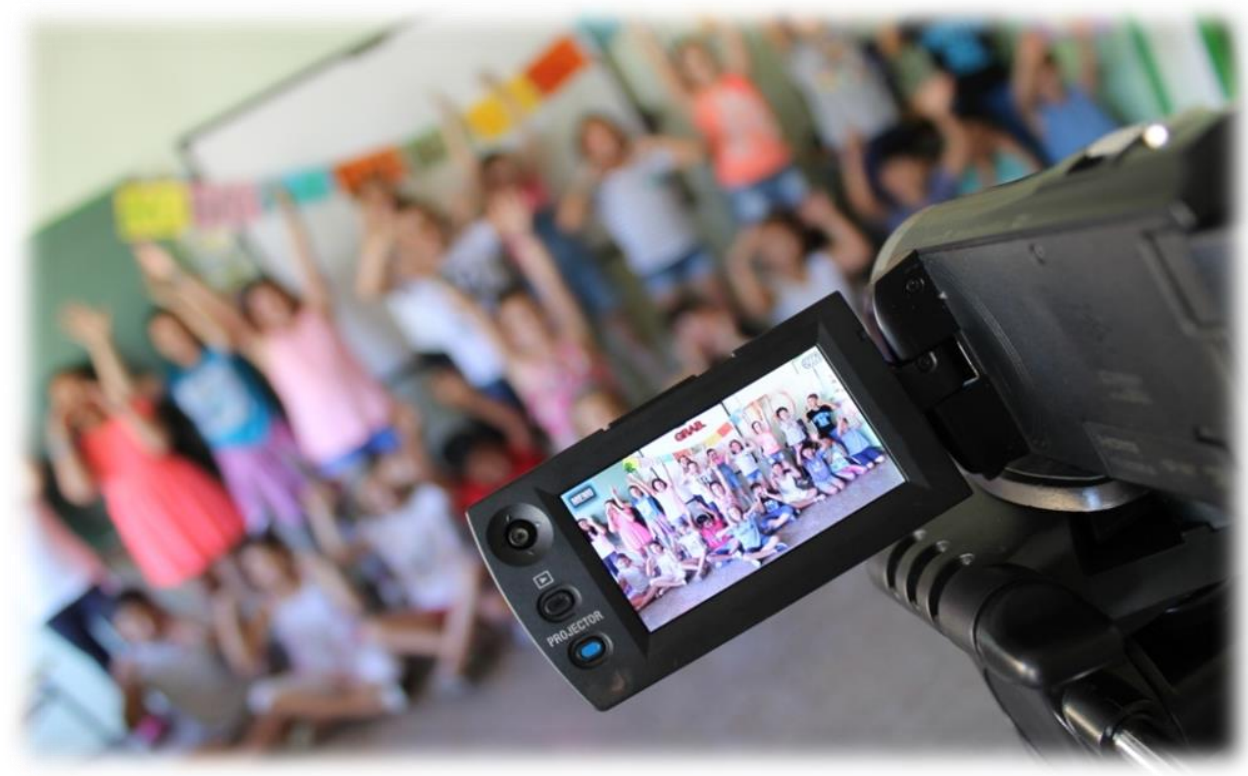

Figura 3. Momento de la grabación del videoclip. Fuente: fotografía propia

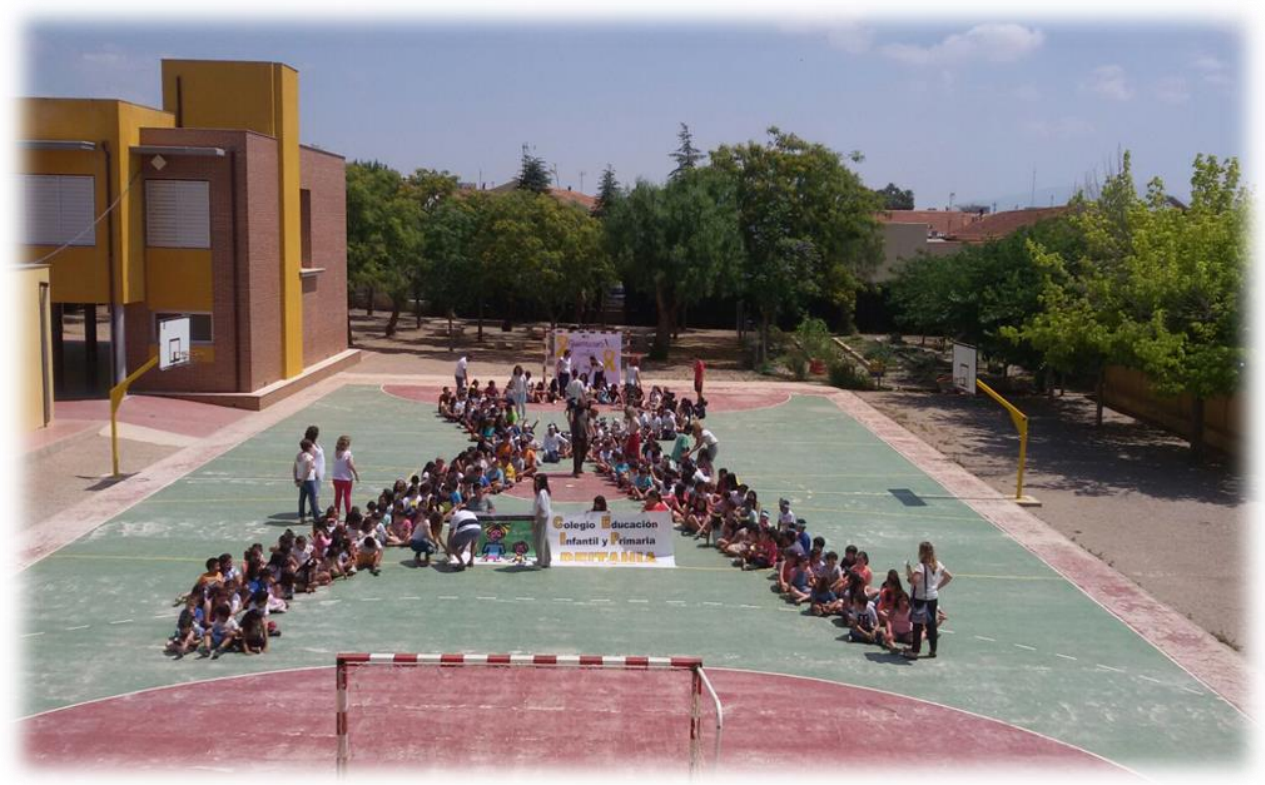

Figura 4. Momento de la grabación del videoclip. Fuente: fotografía propia 


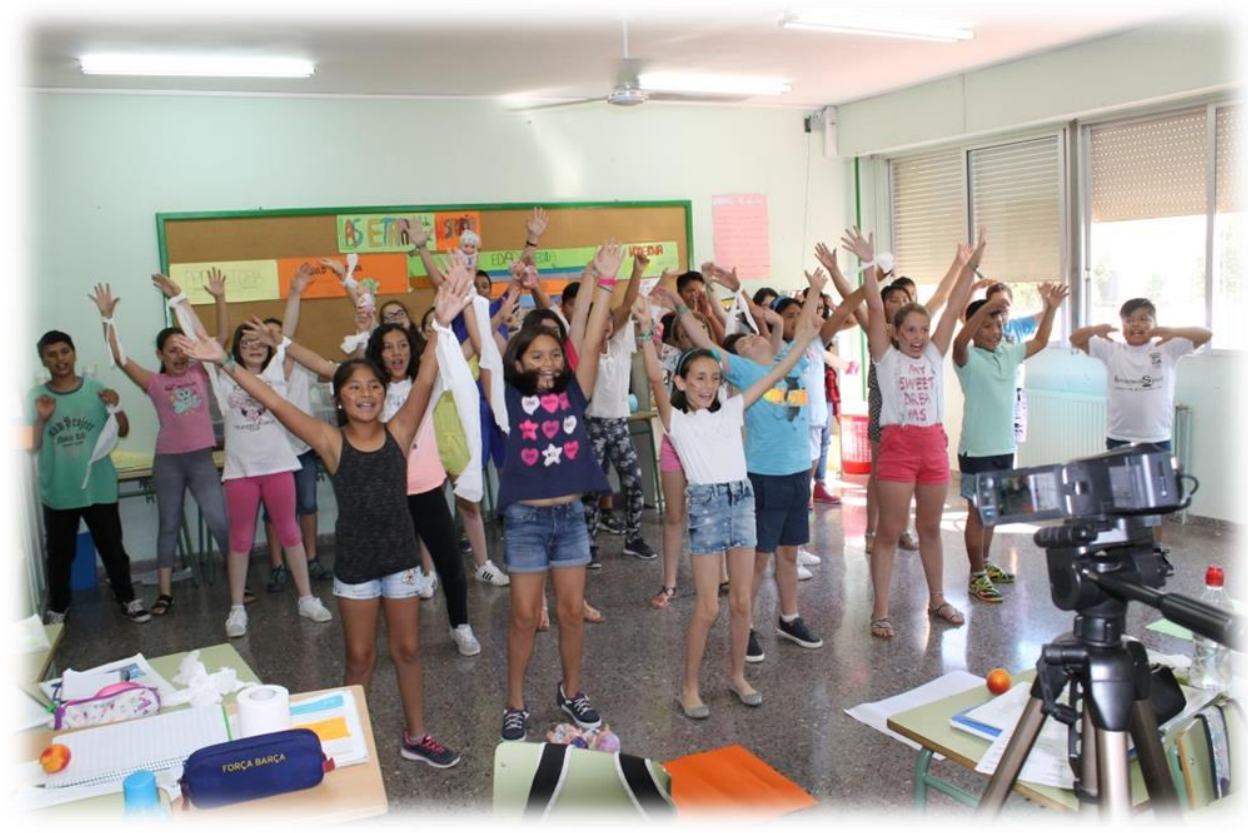

Figura 5. Momento de la grabación del videoclip. Fuente: fotografía propia

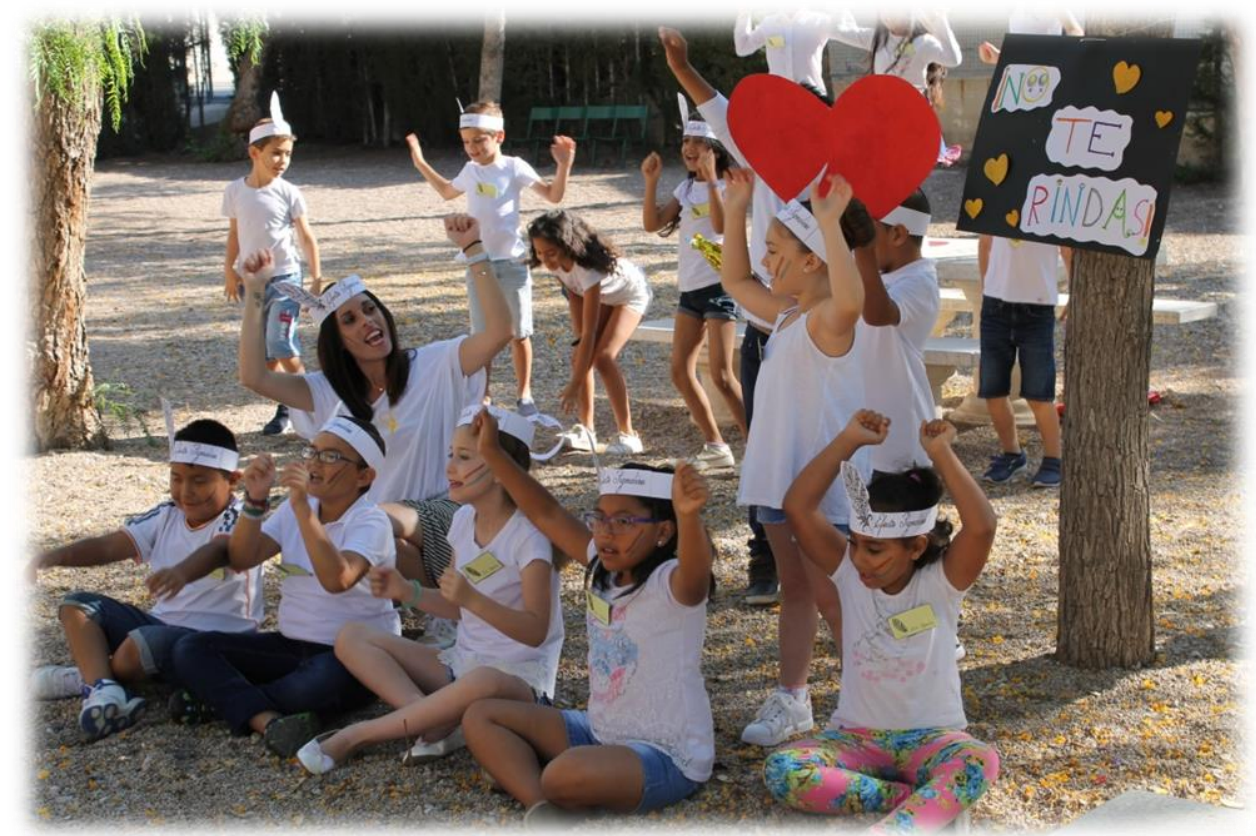

Figura 6. Momento de la grabación del videoclip. Fuente: fotografía propia 


\section{Evaluación del proyecto}

La población a la que se le ofreció el instrumento de evaluación con el que se mediría el nivel de éxito en cuanto al alcance de los objetivos planteados fue de 157 alumnos, cifra que coincide con los escolares participantes en el proyecto. Finalmente, la muestra estuvo compuesta por 142 alumnos.

\subsection{Instrumentos de evaluación}

A cada clase participante de este proyecto a partir de $2^{\circ}$ de primaria se les pasó un breve cuestionario con 6 preguntas dicotómicas y una pregunta con una escala likert de 5 niveles con el fin de conocer la percepción del alumnado sobre este proyecto de aprendizaje servicio. El instrumento de evaluación se puede apreciar en la figura 7:

1. ¿Conocias antes de realizar esta experiencia a AFACMUR?

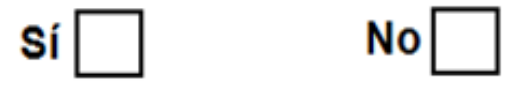

2. ¿Has aprendido contenidos relacionados con el cáncer, como qué es, tratamientos, síntomas, etc.?

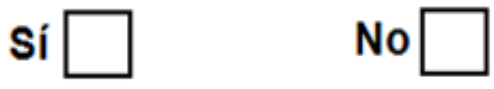

3. ¿Consideras que esta experiencia es una manera óptima para trabajar la educación en valores (solidaridad, generosidad, voluntad, superación....)?

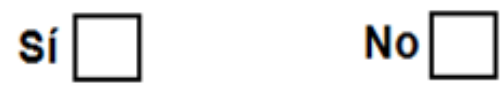

4. ¿Te ha motivado esta experiencia para participar en ella de manera activa?

$\mathrm{S}$

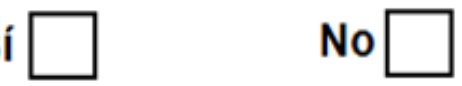

5. ¿Consideras que has fortalecido lazos con el resto de compañeros y con la comunidad educativa en general?

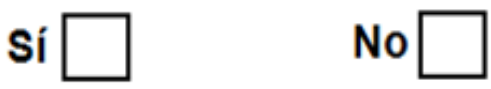

6. ¿Te gustaría que el colegio propusiese otra actividad similar?

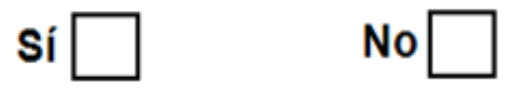

7. Rodea el número que se corresponda con tu satisfacción al haber realizado esta experiencia:

$\begin{array}{ccccc}\mathbf{1} & \mathbf{2} & \mathbf{3} & \mathbf{4} & \mathbf{5} \\ \text { Nada } & \text { Algo } & \text { Satisfecho } & \begin{array}{c}\text { Muy } \\ \text { Satisfecho }\end{array} & \begin{array}{c}\text { Súper } \\ \text { Satisfecho }\end{array} \\ \text { Satisfecho } & \text { Satisfecho } & & & \end{array}$

Figura 7. Cuestionario empleado. Fuente: elaboración propia 


\subsection{Resultados}

\subsubsection{Resultados del instrumento de evaluación}

Para mostrar los resultados de las siete cuestiones se utilizarán diagramas de sectores, de esta forma podremos conocer la frecuencia de respuesta de una manera más rápida y visual. A continuación se muestran las preguntas con sus resultados.

1. ¿Conocías antes de realizar esta experiencia a AFACMUR?

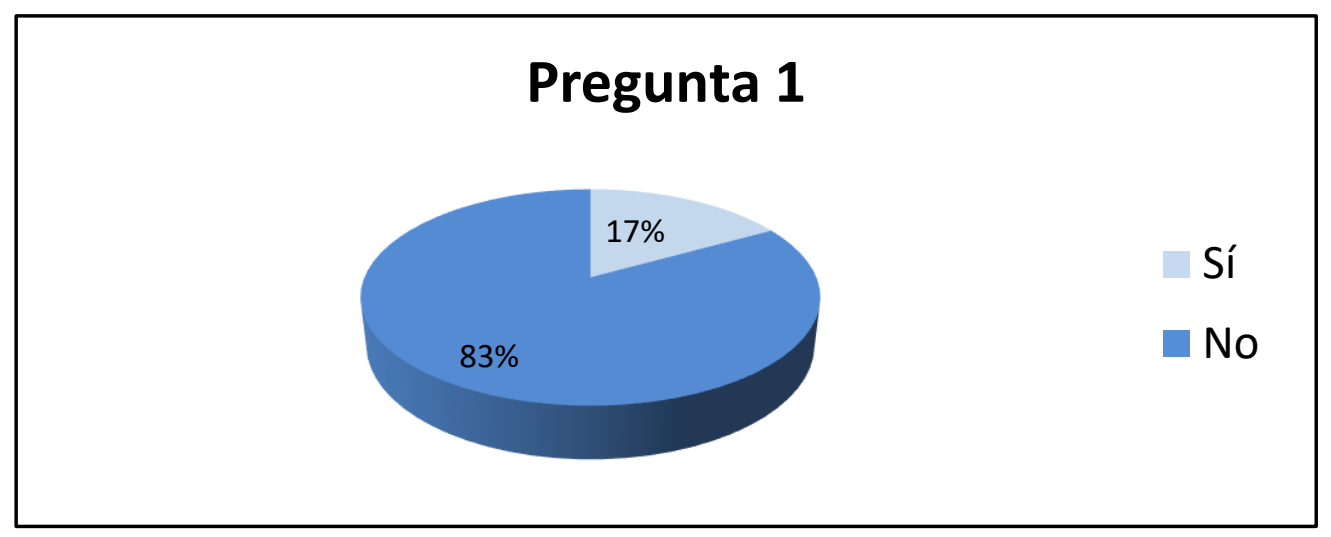

Figura 8. Diagrama de sectores de la pregunta 1. Fuente: elaboración propia

Como bien evidencian estos resultados (figura 8), la gran mayoría de los escolares no conocían la asociación sobre la que se ha basado este proyecto. Es decir, ha sido a raíz del mismo cuando han comenzado a informarse e interesarse por AFACMUR. Señalar que en determinadas ocasiones el alumnado pensaba que AECC (Asociación Española Contra el Cáncer) se encargaba en su totalidad de cubrir las necesidades que estén al alcance de la asociación de los niños oncológicos.

2. ¿Has aprendido contenidos relacionados con el cáncer, como qué es, tratamientos, síntomas, etc.? 


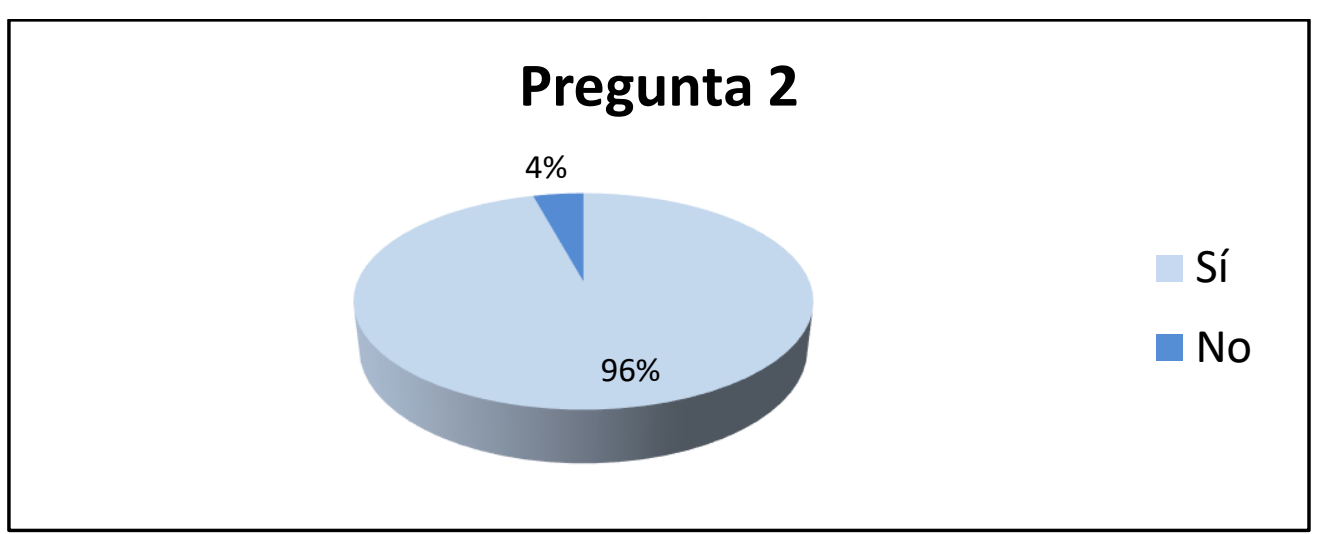

Figura 9. Diagrama de sectores de la pregunta 2. Fuente: elaboración propia

En referencia a esta pregunta y teniendo en cuenta los resultados mostrados en la figura 9 , cabe destacar que aunque a un $4 \%$ les sonaban algunos conceptos relacionados con esta enfermedad, no conocían ciertamente la definición, ejemplos de ello fueron: quimioterapia, leucemia, radioterapia, etc.

3. ¿Consideras que esta experiencia es una manera óptima para trabajar la educación en valores (solidaridad, generosidad, voluntad, superación, entre otros)?

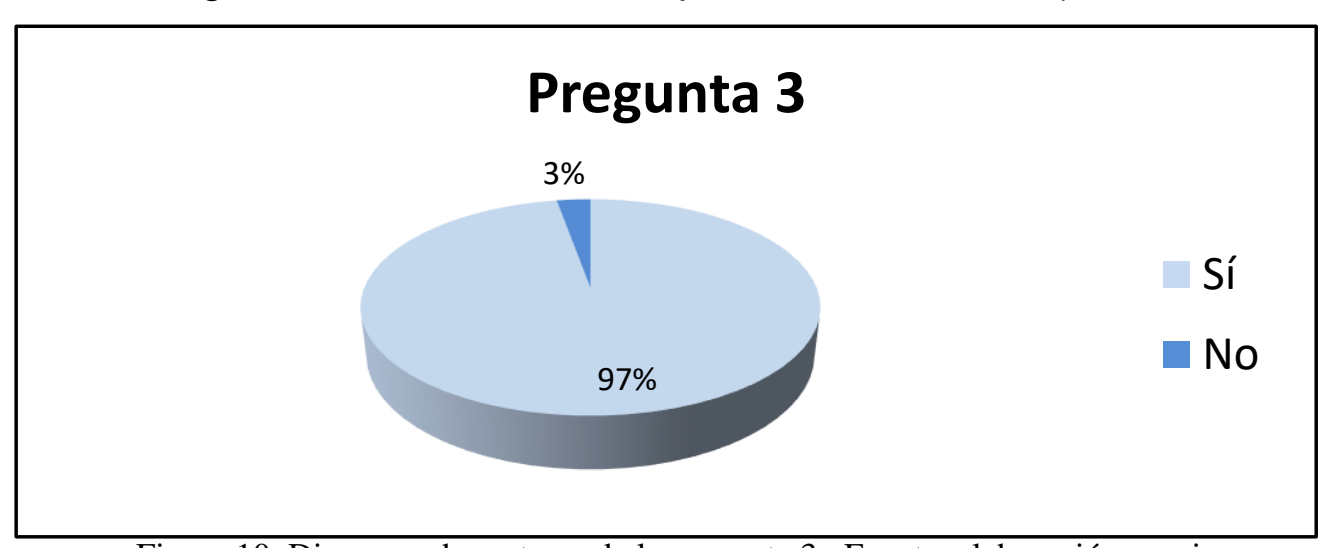

Figura 10. Diagrama de sectores de la pregunta 3. Fuente: elaboración propia

El equipo docente fue el que decidió sobre qué valores se focalizaría con la puesta en práctica de "Meraki, de niños para niños". Una vez realizada la encuesta y teniendo en cuenta la figura 10 , se aprecia cómo queda reflejada en ella la consideración positiva por parte del alumnado sobre la correspondencia entre los valores trabajados y el proyecto llevado a cabo. 
4. ¿Te ha motivado esta experiencia para participar en ella de manera activa?

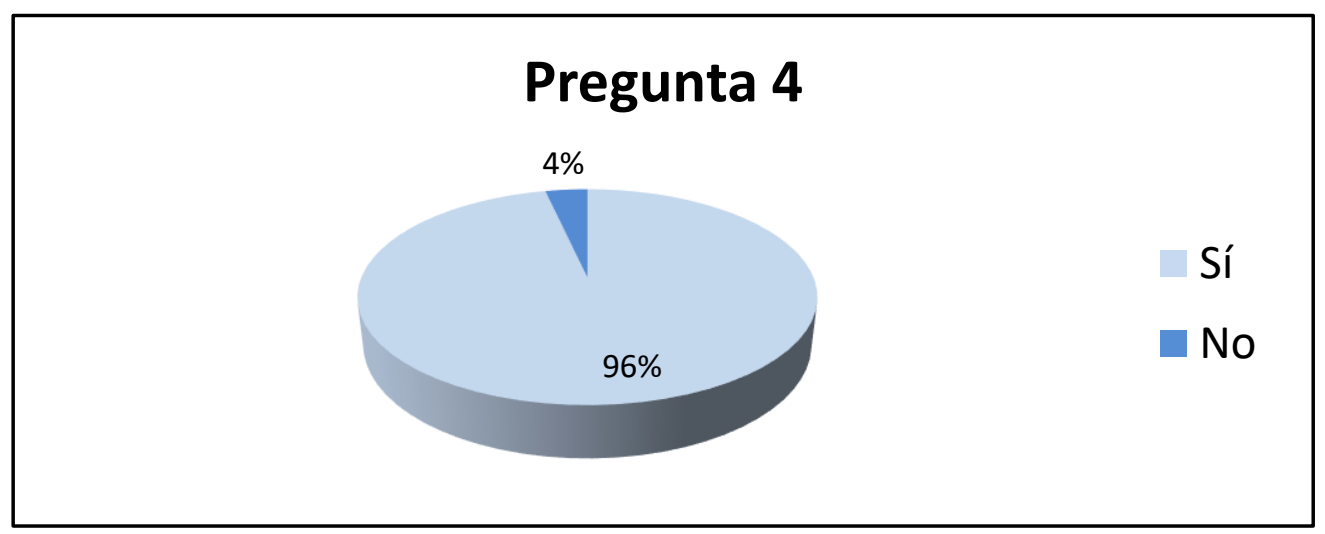

Figura 11. Diagrama de sectores de la pregunta 4. Fuente: elaboración propia

La respuesta afirmativa de esta cuestión no solo se puede ver expuesta en el diagrama de barras de la figura 11, también queda puesta en relieve en los resultados alcanzados con el proyecto. Si el alumnado no hubiese adquirido un compromiso que le hubiese hecho participar en este proyecto de una manera tan activa como lo han hecho, no hubiese sido posible alcanzar los objetivos de la manera que se han conseguido.

Ciertos discentes que optaron por señalar la respuesta negativa de esta pregunta afirmaron que les producía tristeza el escuchar la canción sobre la que se grabaría el videoclip y pensar que lo que estaban haciendo era para los niños oncológicos. No obstante, se hizo hincapié en que una de las finalidades del proyecto en cuanto al alumnado no es generar un estado de tristeza, sino todo lo contrario, ya que estaban aportando toda la ayuda posible por mejorar el bienestar de los niños enfermos.

5. ¿Consideras que has fortalecido lazos con el resto de compañeros y con la comunidad educativa en general? 


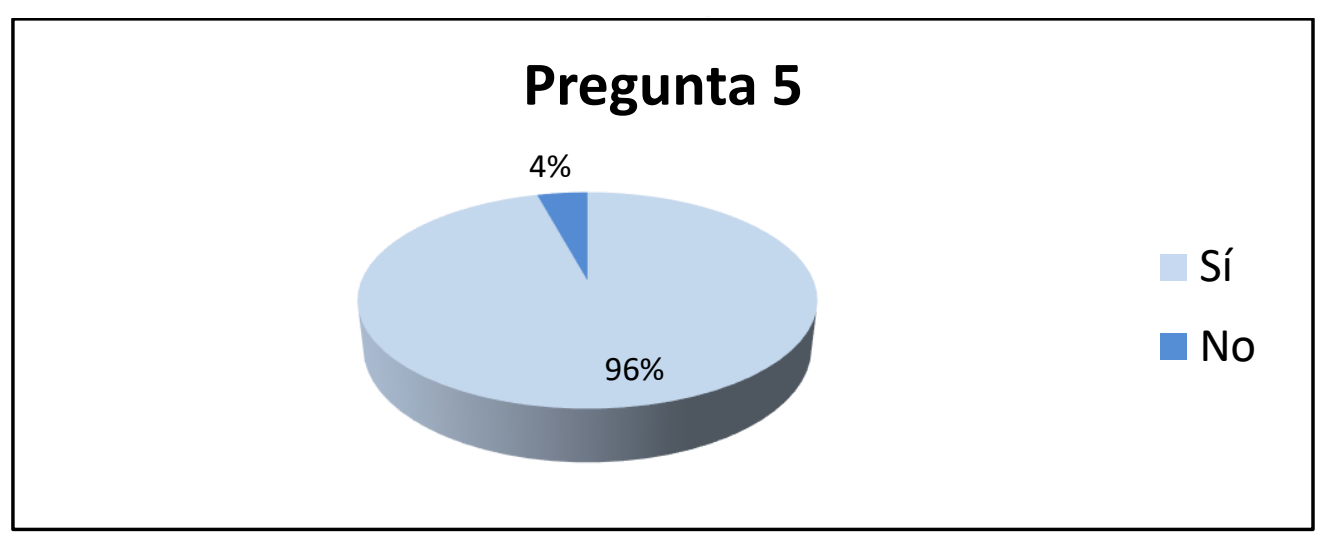

Figura 12. Diagrama de sectores de la pregunta 5. Fuente: elaboración propia

La respuesta afirmativa vuelve a primar en la quinta pregunta, ello queda evidenciado en la figura 12. El alumnado consideró la respuesta afirmativa puesto que el proyecto ha velado en la mayor parte de sus actividades por el trabajo cooperativo y colaborativo (dependiendo de la actividad y cursos). Además, al ser los propios alumnos los que desarrollaron los roles de vendedores de pulseras y entradas, consiguieron vincular aún más a la comunidad educativa con el proyecto llevado a cabo.

6. ¿Te gustaría que el colegio propusiese otra actividad similar?

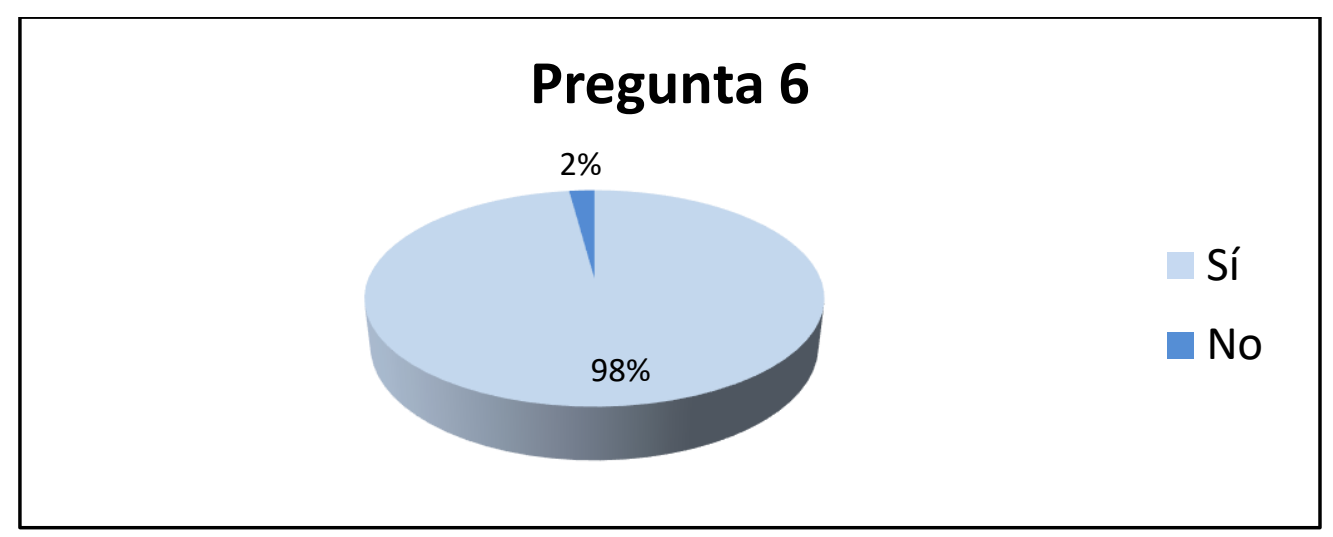

Figura 13. Diagrama de sectores de la pregunta 6. Fuente: elaboración propia

Como bien evidencia la figura 13, los escolares se decantaron en su mayoría por la respuesta afirmativa. Aunque focalizando en el $2 \%$ que ha señalado lo contrario, cabe destacar, como bien justificaron en el cuestionario que no le gustaría que el 
colegio propusiese una actividad similar porque consideraban que sería para la misma asociación y con actividades similares. Por lo que preferían que fuese algo diferente, es decir, un proyecto de aprendizaje servicio solidario pero para una asociación diferente, con otros objetivos y diferentes actividades.

7. Rodea el número que se corresponda con tu satisfacción al haber realizado esta experiencia de "Meraki, de niños para niños"

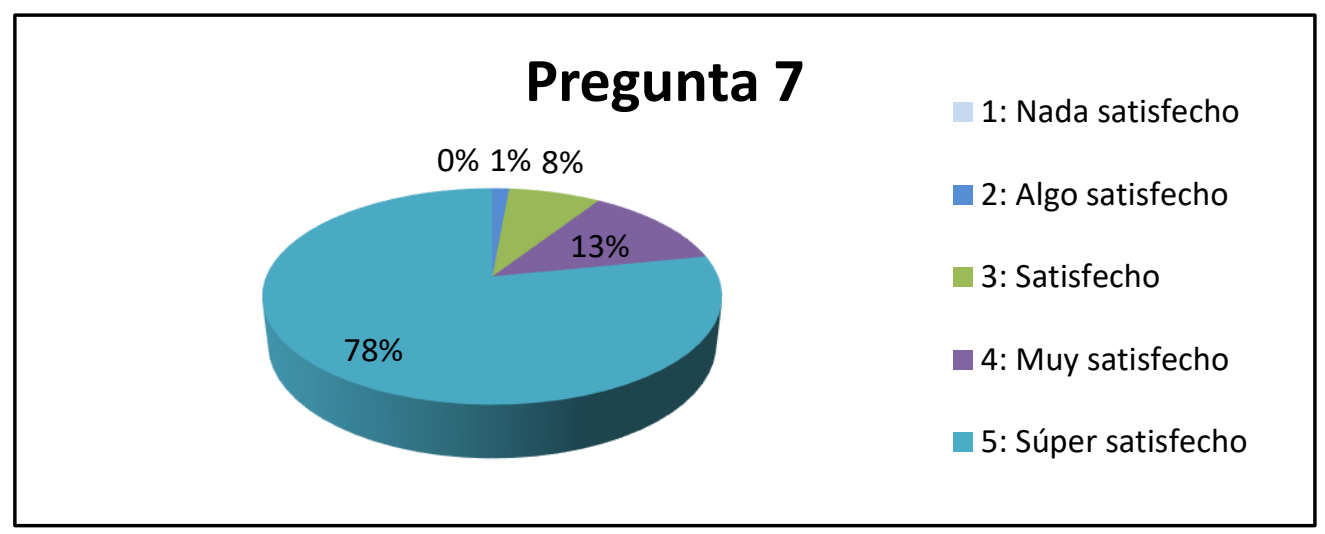

Figura 14. Diagrama de sectores de la pregunta 7. Fuente: elaboración propia

Teniendo en cuenta los resultados que aparecen en la figura 14 y valorando que las opciones 4 (muy satisfecho) y 5 (súper satisfecho) se corresponden con una valoración positiva hacia el proyecto. Cabe destacar el éxito satisfactorio por parte del alumnado al haber desarrollado el presente proyecto, es decir, la amplia mayoría se sintió realizada, satisfecha y con una sensación de no haber malgastado el tiempo.

\subsubsection{Resultados del proyecto llevado a cabo}

Haciendo ahora alusión a los resultados de "Meraki, de niños para niños", señalar que las dos donaciones (fondos económicos para la asociación y aportación anímica a través del videoclip) que se pretendían hacer, se han conseguido y además, superando las expectativas con las que contaba el centro.

En cuanto al dinero recaudado para AFACMUR se pidió a la asociación en un principio unas 50 pulseras, ya que se preveía que no se venderían muchas debido a las situaciones económicas de muchas familias del centro educativo donde se desarrolla 
esta experiencia. Finalmente, se vendieron 200 pulseras. La cantidad equivalente de estas, sumada a lo recaudado de la venta de entradas para el evento solidario y las donaciones libres han sumado un total de $759 €$. Dicha cantidad de dinero fue donada íntegramente a la Asociación de Familiares de niños con Cáncer de Murcia. En la figura 15 se puede apreciar el momento de la comunicación de la cantidad conseguida ante los medios de comunicación regionales. Además, en el enlace web adjunto a continuación (https://goo.gl/jaxc2y) se puede visualizar el reportaje que realizó el canal de televisión 7RM sobre el proyecto desarrollado.

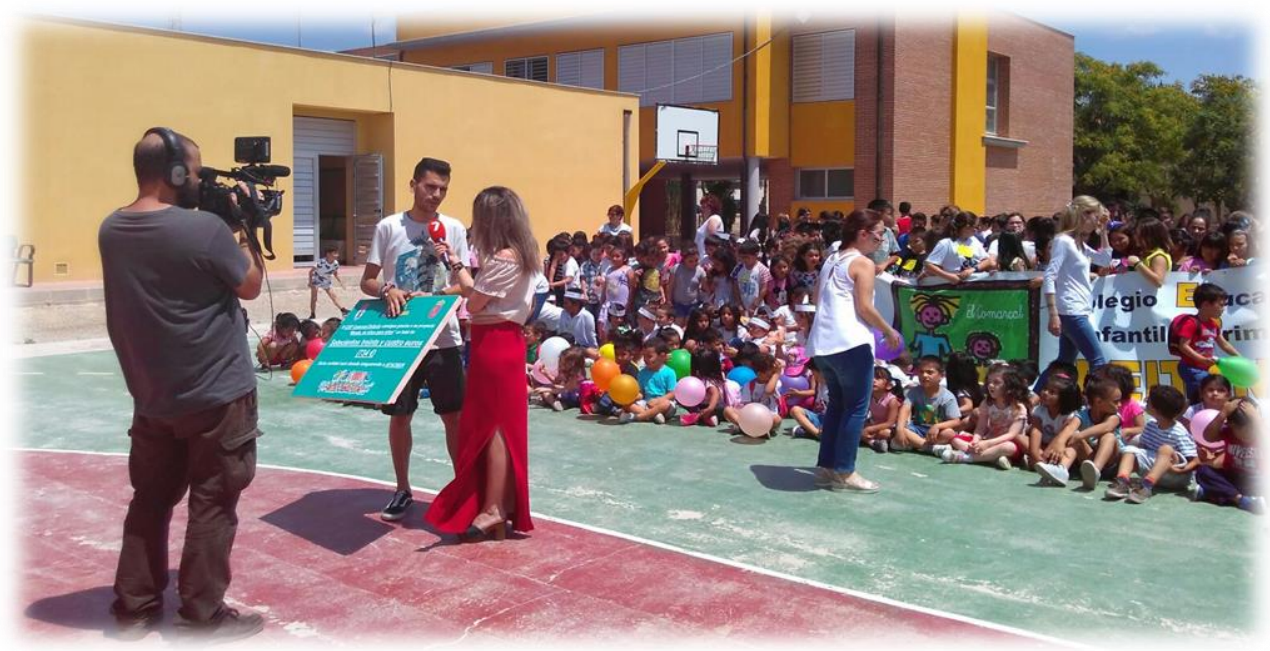

Figura 15. Momento de la comunicación ante el canal de televisión regional (7RM) de la cantidad conseguida. Fuente: fotografía propia

El fin para el que se destinaría el dinero recaudado por el colegio nos indicaron desde la asociación que sería para comprar material, el cual se utiliza todas las tardes en la unidad de oncología infantil del hospital Virgen de la Arrixaca (Murcia) para hacer manualidades, talleres, juegos, etc. Además, parte de la cantidad final también se derivaría a los gastos que conlleva la realización del campamento de verano que realizan los niños que han superado la enfermedad junto a sus hermanos, amigos o familiares menores cercanos.

En cuanto al rodaje del videoclip, la jornada de grabación se desarrolló como estaba prevista, sin ninguna alteración ni dificultad. Por lo tanto, el videoclip también se publicó el día de la fecha estimada y tuvo un alcance que también superó las expectativas, ya que en tan solo 3 días alcanzó en la red social de Facebook las 10000 visualizaciones y en YouTube 1200. El resultado de este se puede apreciar en el 19.2 
siguiente

enlace

web:

https://www.youtube.com/watch?v=YbvQLLUnHmE\&feature=youtu.be

\section{Conclusiones}

Valorando que el presente proyecto ha sido el primero que se ha desarrollado en este centro educativo en cuanto a aprendizaje servicio, cabe destacar que se han cumplido de manera óptima los principales objetivos que se han detallado al inicio de este trabajo y como bien se ha señalado anteriormente, destacar la superación de las expectativas de dicho proyecto.

Teniendo en cuenta algunas de las respuestas negativas aportadas en el cuestionario final referentes a las cuestiones de valoración de lo aprendido del cáncer, sistema inmunológico, etc. Y a la educación en valores, cabe decir que por mínima que haya sido la muestra que ha marcado dicha opción, se ha tenido en cuenta a nivel escolar para realizar de manera más cooperativa la programación y el material didáctico conveniente para cada grupo a nivel de tramo escolar.

No obstante, queda evidenciado en cada una de las gráficas anteriormente mostradas la satisfacción general del alumnado tras la realización de "Meraki, de niños para niños". Además de la utilidad que este ha tenido para ellos, ya que han estado trabajando simultáneamente a nivel teórico, práctico y actitudinal. Siendo por lo tanto una educación holística del alumnado, la cual fomenta el desarrollo competencial para la vida y por la vida de cada uno de ellos, como bien señalaría Decroly. Por lo tanto, coincide plenamente con las afirmaciones que realizan Murphy (2010) y Verjee (2010), ya que valoran que con un proyecto de ApS se da lugar al desarrollo óptimo de las capacidades intelectuales y las habilidades profesionales del alumnado.

Tal ha sido la satisfacción y el compromiso que la comunidad educativa en sí ha adquirido con este proyecto, que teniendo en cuenta la primera pregunta de todas, la cual es para conocer si el alumnado conocía antes de "Meraki, de niños para niños" a AFACMUR. A pesar de que en la gráfica expuesta anteriormente se puede apreciar claramente como la mayoría del alumnado no conocía dicha asociación. Numerosos alumnos y por consiguiente sus familiares, se han interesado por esta asociación y se han ofrecido a inscribirse como voluntarios, hacer donaciones anuales por simbólica 
que pudiese ser, etc.

Por último, debido a la motivación general de toda la comunidad educativa tras la realización de esta experiencia y de los beneficios que los proyectos de ApS pueden aportar. Se están teniendo en cuenta diversas opciones para el próximo curso académico tras consultar las ideas aportadas por Halsted (1998) y otros proyectos ya desarrollados (proyecto ríos, cibermánagers, entre otros).

Tras conocer y analizar los resultados de proyectos como "Meraki, de niños para niños" se evidencian en la práctica educativa los beneficios que aportan al desarrollo holístico y competencial del alumno, convirtiéndose por lo tanto en un ápice de ánimo para desarrollar proyectos similares en los centros escolares.

\section{Referencias bibliográficas}

Batlle, R., Bosch, C., Palos, J., Puig, J. (2007). Aprendizaje servicio: Educar para la ciudadanía. Barcelona: Editorial Octaedro.

Conway, J.M., Amel, E.L. \& Gerwien, D.P. (2009). Teaching and learning in the social context: A meta-analysis of service learning's effects on academic, personal, social, and citizenship outcomes. Teaching of Psychology, 36, 233-245.

Ferrán, A. y Guinot, C. (2012). Aprendizaje-servicio: propuesta metodológica para trabajar competencias. Portularia: Revista de Trabajo Social, 12, 187-195.

Gil, J. (2012). El Aprendizaje-Servicio en la enseñanza superior: una aplicación en el ámbito de la Educación Física (Tesis doctoral). Universidad Jaume I, Castellón.

Halsted, A. (1998). Educación redefinida: la promesa del aprendizaje servicio. En Ministerio de Educación de la Nación (Ed.), El servicio a la comunidad como aprendizaje escolar (pp.23-24). Buenos Aires: Ministerio de Educación de la Nación.

Kendall, J. (1990). Combining Service and Learning: A Resource Book for Community and Public Service. Raleigh: NSIEE.

Martín, C. y Ruiz, M. (1996). La formación del profesor de Educación Infantil y Primaria. En V. García (Ed.), Tratado de Educación Personalizada. Formación de profesores para la educación personalizada (pp.153-186). Madrid: Ediciones Rialp. S.A. 
Martínez, B. (2014). Aprendizaje-Servicio y Educación Inclusiva. Educación y futuro: revista de investigación aplicada y experiencias educativas, 30, 183-208.

Murphy, T. (2010). Conversations on engaged pedagogies, independent thinking skills and active citizenship. Educational Research, 20 (1), 39-46.

Puig, J.M. (Coord.). Aprendizaje Servicio (ApS). Educación y compromiso cívico. Barcelona: Graó.

Puig, J.M. et al. (2011). Aprendizaje-servicio y Educación para la Ciudadanía. Revista de Educación, 1 (n. ำextra), pp. 45-67.

Rodríguez, M., Ordóñez, R. (2015). Una experiencia de aprendizaje-servicio en comunidades de aprendizaje. Profesorado, revista de currículum y formación del profesorado, 19 (1), 314-333.

Rubio, L. (2009). El aprendizaje en el aprendizaje servicio. En J. M. Puig (Coord.), Aprendizaje servicio (APS) Educación y compromiso cívico (pp. 91-106). Barcelona: Graó.

Stanton, T. (1990). Service Learning: Groping Toward a Definition. In J. Kendall (Ed.), Combining Service and Learning: A Resource Book for Community and Public Service (pp. 65-67). Raleigh: NSIEE.

Tapia, M.N. (2007). Aprendizaje y servicio solidario: en el sistema educativo y las organizaciones juveniles. Buenos Aires: Editorial Ciudada Nueva.

Tapia, M.N. (2004). La solidaridad como pedagogía: el Aprendizaje-Servicio en la escuela. Buenos Aires: Ciudad Nueva.

Tapia, M.N. (2013). El modelo pedagógico de Aprendizaje Servicio Solidario (video conferencia. Extraído el 12 de abril de 2017, de https://goo.gl/Ys3FfD

Verjee, B. (2010). Service-learning: Charity-based or transformative? Transformative Dialogues: Teaching \& Learning Journal, 4 (2), 1-13. 


\section{REFERENCIA BIBLIOGRÁFICA}

García Tudela, P.A. (2017). "Meraki, de niños para niños", una experiencia de Aprendizaje Servicio Solidario (ApSS). Aula de Encuentro, 19 (2), pp. 144-169. doi: https://dx.doi.org/10.17561/ae.v19i2.6

\section{Pedro Antonio García Tudela es}

Maestro de Educación Primaria en la región de Murcia

y miembro del grupo de investigación GITE (Grupo de Investigación de Tecnología Educativa) Correo-e: pedro21195@gmail.com o pedroantonio.garcia4@um.es 